ESJ Humanities

\title{
La perception des enseignants et des élèves sur l'examen normalisé et le contrôle continu pour la matière de science de la vie et de la terre
}

\author{
El Janous Youssra \\ Formation Doctorale en ingénierie pédagogie et didactique des \\ sciences.Centre d'Études doctorales sciences et technologies (LIRIP) \\ École Normale Supérieure de Tétouan. Université Abdelmalek Essaâdi, \\ Tétouan, Maroc \\ Laafou Mohamed \\ Jannati-Idrissi Rachid \\ Madrane Mourad \\ Professeur, Laboratoire Interdisciplinaire de Recherche en Ingénierie \\ Pédagogique (LIRIP). École Normale Supérieure de Tétouan, \\ Université Abdelmalek Essaâdi, Tétouan, Maroc
}

Doi:10.19044/esj.2021.v17n20p110

Submitted: 26 January 2021

Accepted: 03 June 2021

Published: 30 June 2021
Copyright 2021 Author(s)

Under Creative Commons BY-NC-ND

4.0 OPEN ACCESS

Cite As:

Youssra E.J., Mohamed L., Rachid J-I. \& Mourad M. (2021). La perception des enseignants et des élèves sur l'examen normalisé et le contrôle continu pour la matière de science de la vie et de la terre. European Scientific Journal, ESJ, 17(20), 110.

https://doi.org/10.19044/esj.2021.v17n20p110

\section{Résumé}

Pour obtenir le certificat d'études primaires, secondaires collégiales ou secondaires qualifiants l'élève doit passer un examen normalisé au niveau régional ou au niveau national, dans les différentes matières enseignées. Dans cet article, nous avons traité les notes de la matière science de la vie et de la terre de l'examen normalisé et de contrôle continu. Cette étude menée auprès de 267 enseignants de cette matière (SVT), répartis sur un nombre important des établissements (secondaire collégiale, secondaire qualifiant), vise à comparer entre les notes du contrôle continu et les notes de l'examen normalisé régional et national, réaliser un diagnostic et une analyse de la réalité des notes obtenues dans la matière de SVT au secondaire collégial ou qualifiant, et identifier les perceptions des enseignants ainsi que des élèves sur les notes obtenues. L'ensemble des résultats de cette recherche permet de 
déterminer le niveau de l'échec scolaire dans cette matière.

Mots clés: Contrôle continu, examen normalisé, science de la vie et de la terre, échec scolaire

\title{
The Perception of Teachers and Students on the National Standardized Examination and Continuous Assessment in Life and Earth Sciences
}

\author{
El Janous Youssra \\ Formation Doctorale en ingénierie pédagogie et didactique des \\ sciences.Centre d'Études doctorales sciences et technologies (LIRIP) \\ École Normale Supérieure de Tétouan. Université Abdelmalek Essaâdi, \\ Tétouan, Maroc \\ Laafou Mohamed \\ Jannati-Idrissi Rachid \\ Madrane Mourad \\ Professeur, Laboratoire Interdisciplinaire de Recherche en Ingénierie \\ Pédagogique (LIRIP). École Normale Supérieure de Tétouan, \\ Université Abdelmalek Essaâdi, Tétouan, Maroc
}

\begin{abstract}
To obtain the primary school, junior high and high school certificate, the student must pass a standardized test at a regional or at a national level in the various taught subjects. In this article, we have covered the life science and earth science subject marks of both the standardized exam and continuous assessment. This study, conducted among 267 teachers of this subject (SVT), spreading over a large number of establishments (junior high school, secondary school), aims to compare the scores of the continuous assessment and the scores of the regional and national standardized examination, to achieve a diagnosis and analysis of the reality of the grades obtained in the SVT subject in college secondary or qualifying secondary, and to identify the perceptions of teachers of this subject as well as students on the obtained grades. All the results of this research allowed us to determine the level of failure in this subject.
\end{abstract}

Keywords: Continuous Control, normalized exam, science of life and earth, school failure 


\section{Introduction}

L'échec scolaire connaît une croissance importante au Maroc, il constitue un indicateur du rendement et de la qualité des systèmes éducatifs nationaux, les élèves et leurs familles, le voient comme une menace dangereuse ou une mauvaise vérité lorsqu'ils l'éprouvent de manière personnelle.

Le terme échec scolaire est une notion récente. En effet en France, au milieu du XXème siècle, peu d'élèves atteignaient un niveau d'études long en durée et plus poussé, tandis qu'un nombre plus important d'élèves se rendaient à école juste pour acquérir des savoirs de bases comme lire, écrire et compter. (CHAUVEAU \& ROGOVAS-CHAUVEAU, 1995).

D'une manière plus spécifique, on va traiter le niveau de l'échec scolaire des élèves concernant la matière de science de la vie et de la terre, pour cela on va comparer les notes de l'examen normalisé national et le contrôle continu de cette matière, et ensuite étudier leurs perceptions sur le décalage existant entre les notes de l'examen régional/national et celles des contrôles continus.

\subsection{Contexte / Problématique :}

$\mathrm{Au}$ Maroc, la problématique d'échec scolaire a connu récemment une ampleur inquiétante. Les dernières réformes du système éducatif ont permet aux élèves de réussir leur année scolaire indifféremment des connaissances et des savoirs retenus. Certes, d'importantes avancées ont été enregistrées concernant la réduction du taux de l'échec scolaire à l'école marocaine, mais les désillusions sont nombreuses, surtout la façon dont laquelle les enseignants et les étudiants aperçoivent cette problématique.

Dans cette étude, nous essayerons de déterminer le niveau de difficulté présent dans l'examen national ou régional par rapport aux examens de contrôle continu, qui mènent à préciser le niveau de l'échec scolaire dans la matière de science de la vie et de la terre, en se basant sur une analyse approfondie de deux questionnaires, l'une traite sur les enseignants et l'autre sur les élèves.

\subsection{Objectif général:}

L'objectif de cet article est de déterminer le niveau de difficulté présent dans l'examen normalisé soit national ou régional par rapport aux évaluations de contrôle continu qui se font au niveau de la classe.

\section{Cadre théorique}

Dans les pays développés, les taux de réussite des élèves dans les différents cycles d'enseignement sont en moyenne de l'ordre de $70 \%$. En revanche, ces taux ne dépassent pas 50\% dans la majorité des pays en voie de développement (Chekour, 2019). Au Maroc, malgré les efforts du Ministère 
de l'Éducation Nationale pour améliorer la performance du système éducatif marocain, la situation actuelle est inquiétante (Ibourk \& Amaghouss, 2015).

L'amélioration de l'apprentissage des disciplines scientifiques est parmi les défis du système d'éducation national (MEN, 2008). Des recherches visant à améliorer l'enseignement de ces disciplines ont montré que la performance du Maroc est aux dernières places par rapport aux autres systèmes éducatifs (MEN, 2014).

Dans le système éducatif marocain, plusieurs facteurs influencent l'acquisition du savoir enseigné. Selon une recherche récente, les élèves sont peu impliqués dans les cours (Rassou et al., 2017). Aussi, les approches pédagogiques utilisées dans les classes sont traditionnelles (Chaali \& Chekour, 2018) et les travaux de laboratoire qui favorise la démarche expérimentale sont utilisés occasionnellement (Lamarti et al., 2009).

Dans une autre perspective, l'évaluation joue un rôle important dans la scolarité de ces élèves dans toutes les matières enseignées (Bénit \& Sarremejane, 2019). Elle permet de déterminer le niveau de maitrise des concepts enseignés. Or, au Maroc on constate qu'il y a un écart entre les résultats des élèves en science de la vie et de la terre dans l'examen national ou régional et les contrôles continus.

\subsection{Comparaison statistique des notes des contrôles continus et de l'examen normalisé obtenues auprès de la direction provinciale}

Pour comparer les notes de contrôle continu et de l'examen normalisé, on a choisi les élèves de la troisième année de secondaire collégial à la province d'Ouezzane comme échantillonnage. Le tableau ci-dessous représente les statiques des moyennes des élèves de la promotion de 2019 à l'examen normalisé et au contrôle continu (les données ont été récupérées à partir de système Massar1), le nombre de ces élèves est de 4759 :

Tableau 1 : Statiques des moyennes des élèves de la promotion de 2019 à l'examen normalisé et au contrôle continu

\begin{tabular}{|c|c|c|}
\hline Moyenne & Examen normalisé & Contrôle continu \\
\hline$[0 ; 01[$ & 18 & 0 \\
\hline$[01 ; 02[$ & 72 & 0 \\
\hline$[02 ; 03[$ & 270 & 0 \\
\hline$[03 ; 04[$ & 563 & 3 \\
\hline$[04 ; 05[$ & 759 & 3 \\
\hline
\end{tabular}

${ }^{1}$ C'est un système en ligne qui permet la saisie et la gestion des notes de contrôles continus et des examens, et aussi il servit à l'informatisation de l'ensemble des informations des enseignants, la gestion des emplois du temps et le suivi des retards, absences et sanctions des élèves. Les administrateurs au niveau de la direction provinciale ayant le droit de télécharger et d'exploiter ces données. 


\begin{tabular}{|c|c|c|}
\hline Moyenne & Examen normalisé & Contrôle continu \\
\hline$[05 ; 06[$ & 816 & 13 \\
\hline$[06 ; 07[$ & 663 & 49 \\
\hline$[07 ; 08[$ & 505 & 186 \\
\hline$[08 ; 09[$ & 291 & 540 \\
\hline$[09 ; 10[$ & 231 & 866 \\
\hline$[10 ; 11[$ & 149 & 920 \\
\hline$[11 ; 12[$ & 108 & 682 \\
\hline$[12 ; 13[$ & 77 & 453 \\
\hline$[13 ; 14[$ & 67 & 292 \\
\hline$[14 ; 15[$ & 61 & 233 \\
\hline$[15 ; 16[$ & 50 & 195 \\
\hline$[16 ; 17[$ & 33 & 136 \\
\hline$[17 ; 18[$ & 15 & 112 \\
\hline$[18 ; 19[$ & 9 & 61 \\
\hline$[19 ; 20[$ & 2 & 15 \\
\hline
\end{tabular}

À partir du tableau, on peut bien voir que le nombre des élèves ayant obtenu une moyenne inclue dans l'intervalle [0 ; 01 [ à l'examen normalisé est de 18 élèves, pendant que personne n'a eu cette moyenne au contrôle continu. D'autre part, on peut aussi voir que seulement 2 élèves ont pu atteindre une moyenne dans l'intervalle $[19 ; 20$ tandis qu'on trouve 15 élèves au niveau du contrôle continu.

Pour bien détailler cet effet, nous avons transformé les données du tableau sous forme de graphe. 


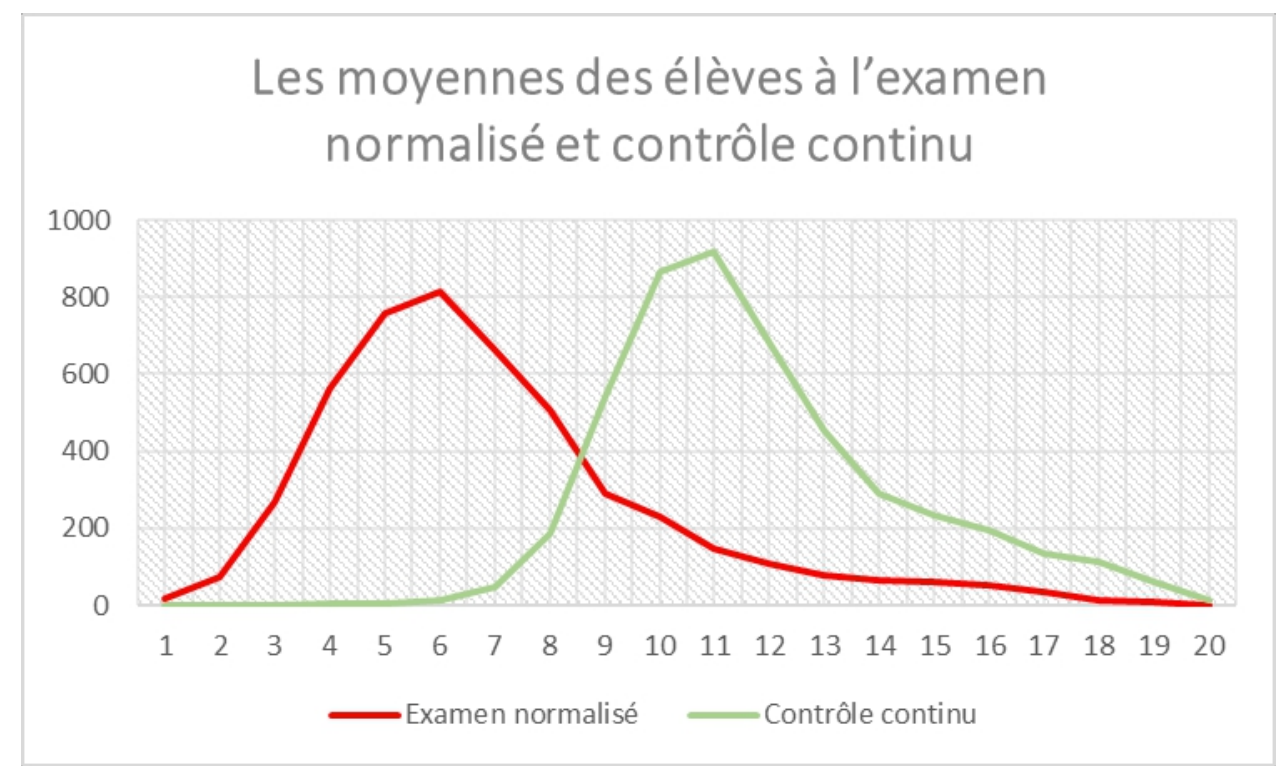

Fig. 1 : Les moyennes des élèves à l'examen normalisé et au contrôle continu

Ce graphe représente deux courbes :

1. La première courbe, en couleur rouge, représente l'évolution du nombre des élèves en fonction de la moyenne (note sur vingt) de l'examen normalisé.

2. La deuxième courbe, en couleur verte représente l'évolution du nombre des élèves en fonction de la moyenne (note sur vingt) du contrôle continu.

À partir de ces courbes, il clair que les notes du contrôle continu sont presque toujours supérieures ou égales à la note $08 / 20$, contrairement à l'examen normalisé où la concentration des nombres d'étudiants est plus élevée dans la région des notes inférieures à 08/20.

À partir de ces deux courbes, nous remarquons que les notes du contrôle continu sont clairement supérieures aux notes de l'examen normalisé.

\section{Méthodologie de la recherche}

\subsection{Choix d'outils}

Parmi les étapes essentielles dans un processus de recherche, on trouve le questionnement. Raison pour laquelle nous avons opté pour ce dernier afin de vérifier la validité des hypothèses que nous avons extrait à partir de l'analyse des bases de données étudiées. Le choix d'un questionnaire comme outil méthodologique de la recherche a pour objectif de :

- Se procurer des réponses qualitatives.

- Faciliter l'accès à l'information. 
- Avoir un grand nombre de personnes et en assez peu de temps.

- Être rempli de manière anonyme, et au rythme de la personne qui répond sans ressentir de pression extérieure ou sans interférence de l'enquêteur.

\subsection{Public cible}

Les participants aux questionnaires sont 267 enseignants d'enseignement secondaire collégial et qualifiant, qui relèvent tous de la direction provinciale d'Ouezzane, et 500 élèves de l'enseignement collégial relevant de différents établissements collégiaux de ladite province.

\subsection{Les questions du questionnaire}

Les questions de ce questionnaire permettent de valider ou d'annuler les hypothèses en postulant que les notes de contrôle continu sont toujours supérieures aux notes de l'examen régional et de l'examen normalisé local.

$\mathrm{Ce}$ questionnaire se compose de quatre questions destinées pour les enseignants :

1. Expliquez-vous les questions le jour de l'examen régional/national aux élèves?

2. Comment trouvez-vous le niveau des questions de l'examen régional/national de la matière que vous enseignez par rapport au niveau des élèves ?

3. Quels sont les critères qui prennent en charge les notes du contrôle continu?

4. Comment procédez-vous pour évaluer vos élèves ?

Le deuxième questionnaire est destiné aux élèves, en se rapportant au contrôle continu et l'examen normalisé, ainsi nous avons posé les questions suivantes aux élèves :

1. Est-ce que l'évaluation à la totalité du programme lors de l'examen normalisé est efficace?

2. Comment trouvez-vous le niveau des questions des examens normalisés par rapport au contrôle continu ?

3. Est-ce que le climat de l'examen influence votre état psychologique ?

4. Comment trouvez-vous les notes du contrôle continu ? 


\section{Résultat et discussion}

\section{Questionnaire 1/ Question 1 : Vous expliquez les questions le jour de l'examen régional/national aux élèves?}

\section{L'explication des questions le jour de l'examen}

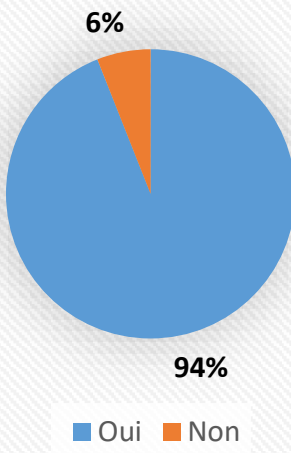

Fig. 2 : L'explication des questions le jour de l'examen

Le graphe représente le pourcentage d'explication des questions le jour de l'examen normalisé national ou régional par les enseignants.

À partir du graphe, 94\% des enseignants n'expliquent pas les questions le jour de l'examen normalisé régional ou national alors que seulement $6 \%$ des enseignants les expliquent.

Nous avons posé cette question pour vérifier l'hypothèse qui annonce que l'absence de l'explication des questions le jour de l'examen régional joue rôle important dans la baisse des notes des examens par rapport au contrôle continue et grâce à ces pourcentages, cette hypothèse a été validée.

Par ailleurs, et afin d'éviter tout décalage entre les notes de l'examen et de contrôle continu il faudra exclure l'explication des questions des devoirs surveillés. 
Questionnaire 1/ Question 2 : Comment trouvez-vous le niveau des questions de l'examen régional/national de la matière que vous enseignez par rapport au niveau des élèves ?

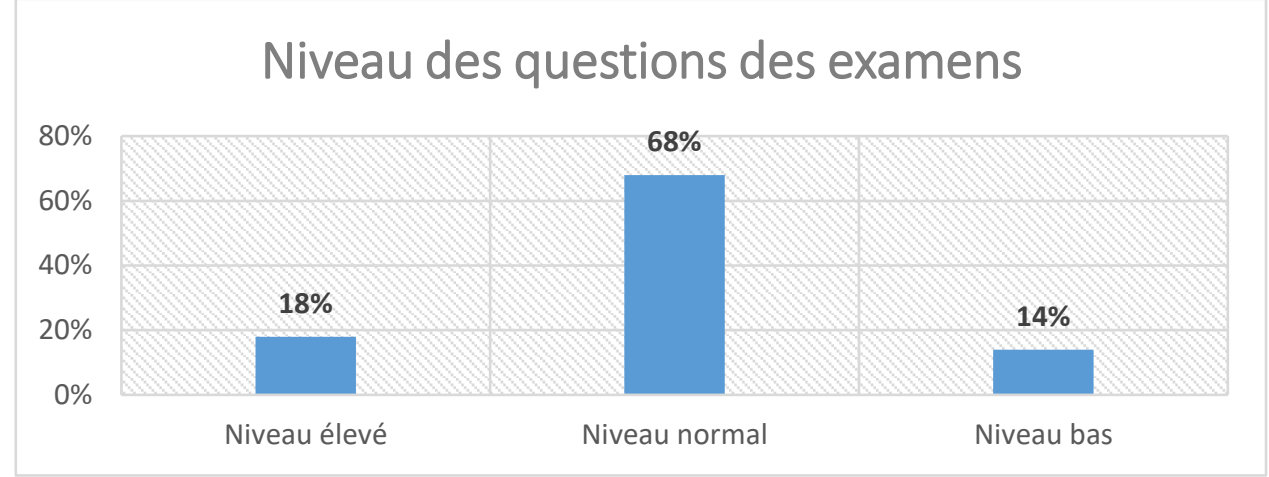

Fig. 3 : Niveau des questions des examens par rapport au niveau des élèves

Le graphe présente les réponses des enseignants à la question qui détermine le niveau des questions de l'examen par rapport au niveau des élèves.

Il en ressort que $68 \%$ des enseignants affirment que le niveau des questions de l'examen normalisé est adapté au niveau des élèves, $18 \%$ des enseignants confirment que le niveau des questions de l'examen est supérieur au niveau des élèves, et $14 \%$ des enseignants disent que le niveau des élèves est plus élevé à celui des questions posées à l'examen normalisé.

À suite de cette question, l'hypothèse qui suppose que le niveau des questions de l'examen est plus élevé que le niveau des élèves, a été annulée.

\section{Questionnaire 1/ Question 3 : Les notes du contrôle continu prises en considération :}

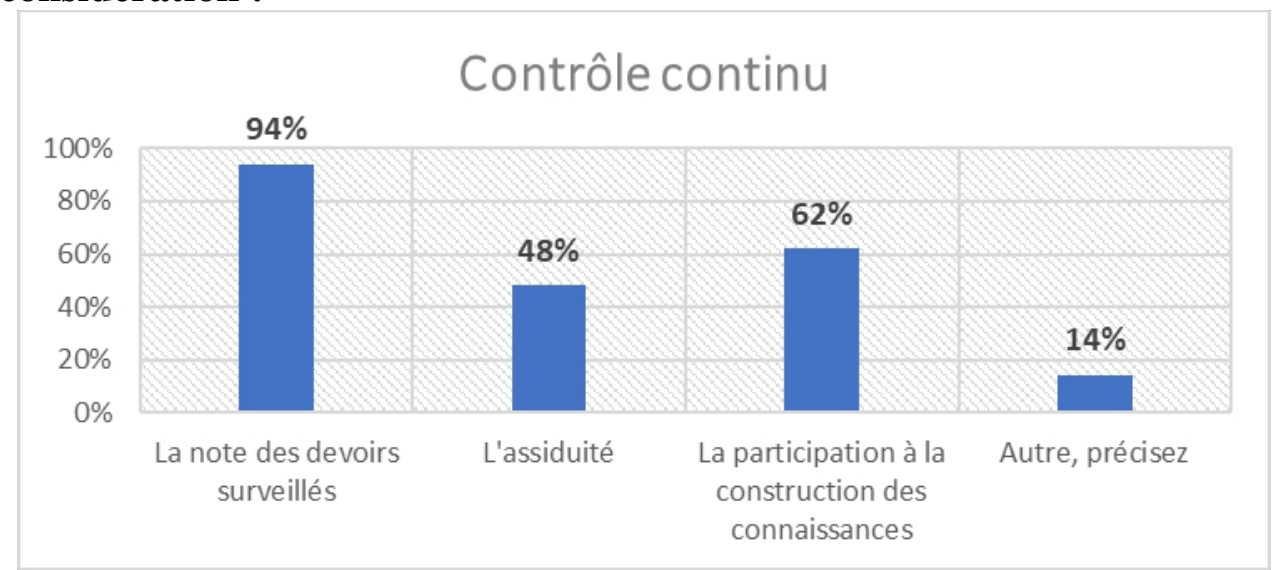

Fig. 4 : Les critères pris en considération lors du calcul du contrôle continu 
Ce graphe représente les critères que les enseignants adoptent dans le calcul de la moyenne du contrôle continu

À partir de ces résultats, il résulte que les enseignants prennent en considération plusieurs critères lors du calcul de la moyenne du contrôle continu d'un élève. En plus des notes des devoirs surveillés, $62 \%$ des enseignants aoutent la participation des élèves à la construction des connaissances, prise en compte comme un critère lors du calcul de la moyenne du contrôle continu, tandis que $48 \%$ des enseignants considèrent l'assiduité des élèves comme une épreuve, $14 \%$ prennent d'autres critères tels que la correction des cahiers, l'absence, la réalisation des devoirs, le comportement de l'élève, la préparation des cours d'avant, la réalisation des recherches et des activités à la maisons.

Nous déduisons qu'à l'aide de ces critères, les notes du contrôle continu, se révèlent généralement, supérieures aux notes de l'examen normalisé.

Questionnaire 1 / Question 4 : Comment procédez-vous à l'évaluation vos élèves ?

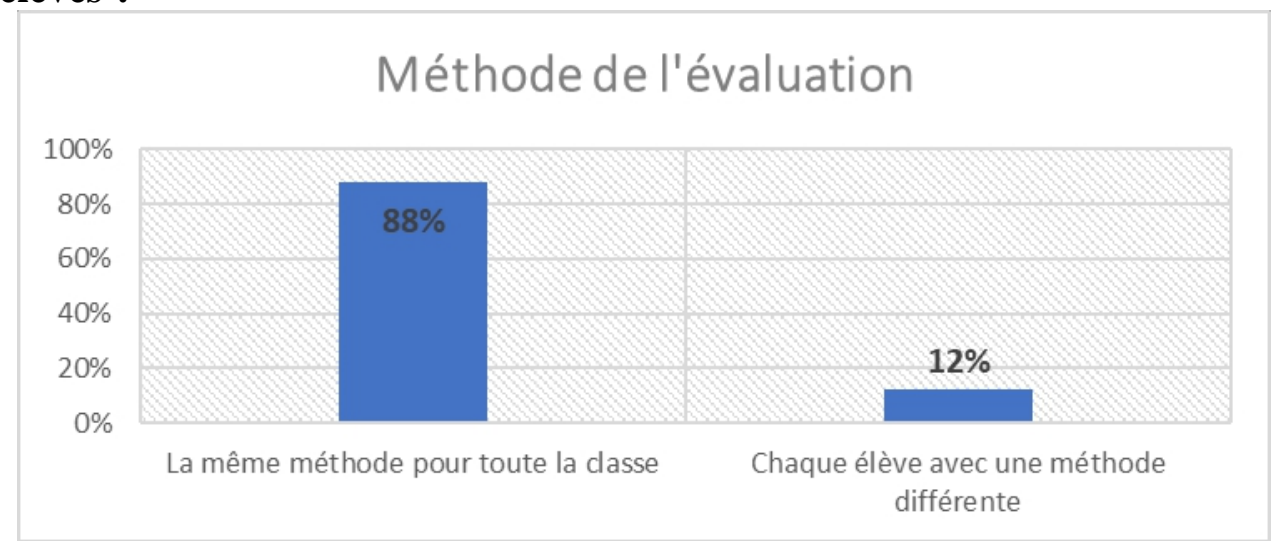

Fig. 5 : Les méthodes d'évaluation

Ce graphe représente la procédure suivie par les enseignants afin d'évaluer leurs élèves.

$88 \%$ des enseignants utilisent la même méthode pour évaluer leurs élèves, et $12 \%$ des enseignants adoptent des méthodes différentes afin d'évaluer les élèves de la même classe.

Autrement dit, $88 \%$ des enseignants n'appliquent pas la pédagogie différenciée (la pédagogie Freinet) lors de l'évaluation des élèves.

La pédagogie différenciée est le fait que chaque élève soit évalué d'une manière particulière, elle est nécessaire à la contribution au développement de la réussite scolaire. Cela explique l'avantage de la pédagogie Freinet. En effet, l'individualisation des apprentissages permet aux élèves qui ont des obstacles de se développer à leurs rythmes, par rapport à leurs buts personnels. 
L'utilisation du même modèle d'évaluation avec tous les élèves est une méthode qui considère la classe comme un groupe cohérent. Ce qui n'est jamais le cas. Le fait d'évaluer chaque élève d'une manière différente et selon son propre profil reste le plus efficace à la réussite scolaire.

Questionnaire 2 / Question 1: Est-ce que l'évaluation à la totalité du programme lors de l'examen normalisé est efficace?

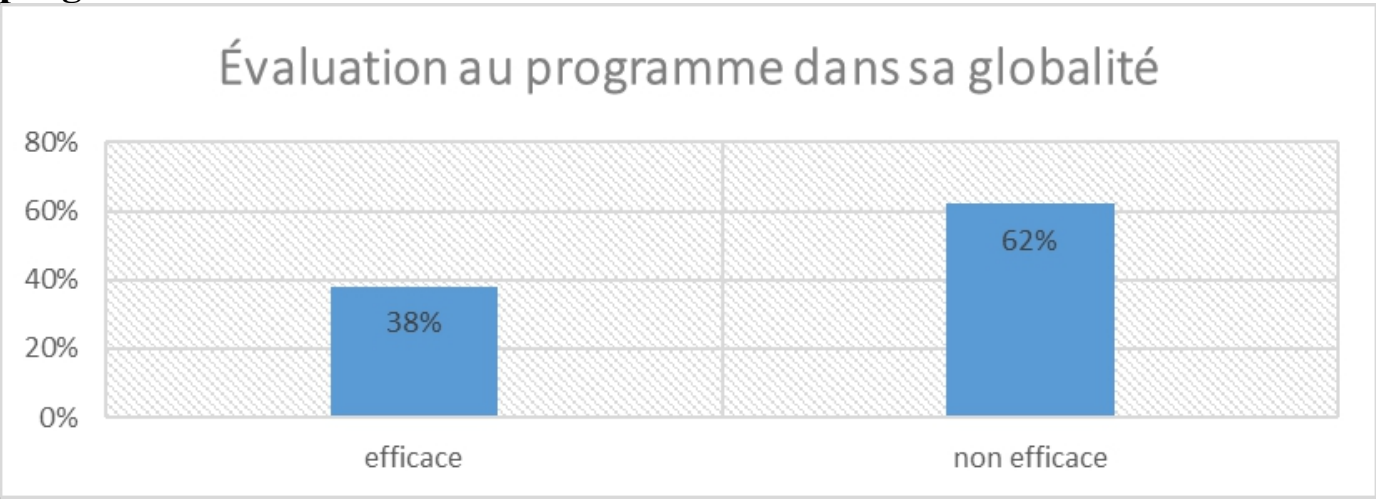

Fig. 6 : L'évaluation du programme dans sa globalité lors des examens normalisés

$62 \%$ des élèves ont déclaré que l'évaluation de la globalité du programme des examens normalisés n'est pas efficace, et $38 \%$ des élèves ont dit le contraire.

Nous concluons que l'évaluation du programme dans sa globalité produit un échec scolaire chez les élèves, car ces derniers refusent d'être évalués dans la totalité du programme d'une matière spécifique.

Par conséquent, il faudra changer cette méthode d'évaluation de l'examen normalisé vu qu'elle est non appréciée par l'élève. Raison pour laquelle les notes du contrôle continu apparaissent plus élevées, par rapport aux notes des examens normalisés.

Questionnaire 2 / Question 2 : Comment trouvez-vous le niveau des questions des examens normalisés par rapport au contrôle continu ?

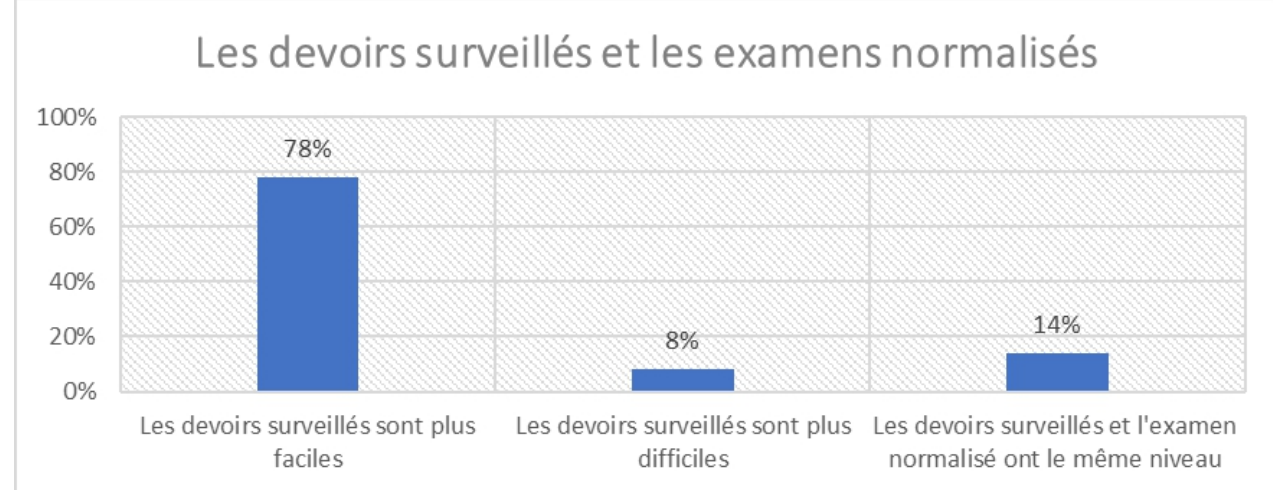

Fig. 7 : L'évaluation de la totalité du programme des examens normalisés 
Ce graphe représente le niveau de difficulté des questions posées dans l'examen normalisé par rapport aux questions posées dans les devoirs surveillés.

À partir de ce graphe, les résultats sont classés comme suit :

1. 78\% des élèves affirment que les devoirs surveillés sont plus faciles que l'examen normalisé.

2. $14 \%$ des élèves disent que les devoirs surveillés et l'examen normalisé ont le même niveau.

3. $8 \%$ des élèves voient que les questions posées à l'examen normalisé sont plus faciles que celles posées dans les devoirs surveillés.

D'après ces résultats, nous constatons que les devoirs surveillés ne respectent pas les normes strictes et les critères d'une évaluation sommative. Pour cela, les élèves considèrent que les devoirs surveillés sont plus faciles que les examens normalisés. Sans oublier que dans les devoirs surveillés, on n'évalue qu'une partie précise du programme scolaire de la matière, contrairement à l'examen normalisé, où on évalue la totalité du programme, ce qui explique la facilité devoirs surveillés.

Alors, il faut sensibiliser les enseignants au respect des méthodes et des règles dans les évaluations afin d'éviter la différence entre les deux. Il ne faut pas oublier aussi que les devoirs du contrôle continu ont pour objectif de préparer les élèves aux examens certifiant. De ce fait, si les enseignants respectent les normes, les notes des élèves aux examens certifiés vont s'améliorer.

Questionnaire 2 / Question 3 : Est-ce que le climat de l'examen influence sur votre psychologie?

L'influence des examens normalisés sur la psychologie

$$
\text { de l'élève }
$$

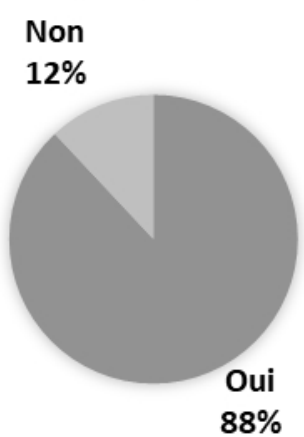

Fig. 8 : La relation entre les examens normalisés et la psychologie de l'élève

Le graphe représente la relation entre la psychologie de l'élève et le climat des examens normalisés. 
À partir de ce graphe, $88 \%$ des élèves disent que l'examen normalisé influence négativement leur psychologie, et seulement $12 \%$ des élèves affirment le contraire.

D'après les réponses des élèves et de tout ce qui est reconnu par les enseignants et les psychologues; l'inquiétude, le trac, et l'angoisse sont classifiés parmi les causes les plus dominantes de l'échec aux examens.

Le trac étant le premier adversaire de l'élève, car il perturbe son intelligence et son imagination. Le trac se déclenche suite à un sentiment d'inquiétude et de peur continue. Au temps de l'examen, ce phénomène peut générer des maux conséquences sur le succès et le résultat de l'élève.

Donc, il s'avère que le climat de l'examen normalisé influence effectivement les résultats scolaires des élèves au collège.

Questionnaire 2 / Question 4 : Comment trouvez-vous les notes de contrôle continu?

\section{Les notes de contrôle continu}

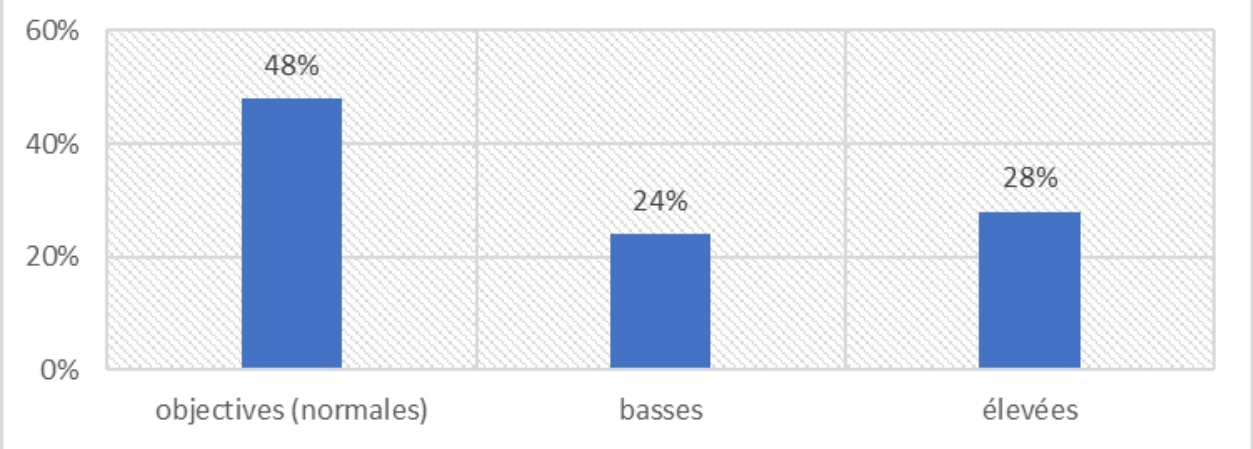

Fig. 9 : Les notes de contrôle continu

Le graphe représente les avis des élèves concernant leurs notes obtenues du contrôle continu.

À partir du graphe, $48 \%$ des élèves constatent que les notes du contrôle continu sont objectives (normales), $28 \%$ des élèves disent que ces notes sont élevées, et $24 \%$ des élèves trouvent qu'elles sont faibles.

Donc, presque la moitié des élèves affirment que les enseignants sont objectifs, et qu'ils donnent à chaque élève la note qu'il mérite.

Pour conclure, notre hypothèse qui annonce que les enseignants gonflent les notes est plus ou moins confirmée.

\section{Conclusion}

Les résultats obtenus visent à diagnostiquer les notes obtenues par les élèves dans la matière de SVT à l'enseignement secondaire dans le contexte éducatif marocain, selon la conception des enseignants eux-mêmes, le 
principal facteur qui mène à ces résultats est le contenu trop chargé de la matière des sciences de la vie et de la terre.

Nos résultats montrent que le climat de l'examen normalisé a une véritable influence sur les résultats scolaires des élèves au collégial. Dans le même contexte, on déduit aussi que pour éviter l'écart entre les notes de l'examen et celles de contrôle continu, il faut éviter l'explication des questions concernant les devoirs surveillés.

Parallèlement aux facteurs qui peuvent dissuader, il on existe des facteurs élequents telle que la méthode d'évaluation à l'examen normalisé considérée déagréable pour la plupart des élèves. Raison pour laquelle, les notes du contrôle continu sont généralement plus élevées par rapport aux notes des examens normalisés.

Enfin, il nous semble très pertinent de poursuivre des recherches dans ce domaine, pour aboutir à des solutions efficaces permettant d'aider les élèves à s'améliorer pour mieux passer leur examen normalisé.

\section{References:}

1. Bajos S., (2012). Débat : pour ou contre les devoirs à la maison. Disponible sur le lien http://www.leparisien.fr/magazine/grandangle/debat-pour-ou-contre-les-devoirs-a-la-maison-16-10-2012 2237913.php [Consulté le 08 mai 2020]

2. Bénit, S., \& Sarremejane, P. (2019). Les pratiques évaluatives à la maternelle: Des expériences enfantines différenciées en fonction du statut scolaire. Carrefours de l'éducation, 2, 91-106.

3. Chaali, R., \& Chekour, M. (2018). Utilisation de la carte conceptuelle dans le processus d'enseignement apprentissage des sciences de la vie et de la terre. info-crde, 21.

4. Chauveau, G., \& Rogovas-Chauveau, É. (1995). À l'école des banlieues. Esf Editeur.

5. Chekour, M. (2019). Contribution à l'amélioration d'acquisition de concepts en électricité chez les lycéens marocains [Université Abdelamalek Essaadi]. https://doi.org/10.13140/RG.2.2.19550.36160

6. Eljanous, Y. (2016). Echec scolaire, études, analyse, cause et solutions technologiques : Cas de la province d'Ouezzane [Mémoire de Master Spécialisé en Technologies éducatives].

7. Ibourk, A., \& Amaghouss, J. (2015). Le système de formation marocain : Source de dynamisme ou d'inertie? La formation dans tous ses états dans les pays du Maghreb, 23(1), 117-138.

8. Lamarti, L., Ben-Bouziane, A., Akrim, H., \& Talbi, M. (2009). La sortie de terrain: Quelle place et quel rôle dans une démarche scientifique? 
9. MEN. (2008). Programme d'urgence. Rabat: Gouvernement du Maroc.

10. MEN. (2014). Indicateur de l'éducation. Direction de la Stratégie, des Statistiques et de la Planification Division des Etudes et des Statistiques.

11. Rassou, K. K., Khiri, F., Benbrahim, M., Tamraoui, Y., Elberrani, H., \& Anfour, M. (2017). Difficultés Relatives A L'enseignementApprentissage De La Géologie En Classes Secondaires Qualifiantes Cas De La Délégation d'Inzegane Ait Melloul. European Scientific Journal, ESJ, 13, 18. 